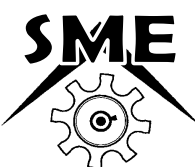

ESTD 2005

\title{
IMPROVING SERVICE QUALITY OF DHABA WITH THE HELP OF QUEUING THEORY
}

\author{
*Kajal Garg and Umesh Sharma
}

${ }^{1}$ Department of Basic and Applied Science, Sanskriti University, Mathura, Uttar Pradesh-281401, India

\begin{abstract}
Queuing theory is the mathematics of waiting lines. It is beneficial in predicting and evaluating system performance. Queuing theory has been used for operations research, manufacturing and systems analysis. The common problem in almost every famous Dhaba is that they lose their customers due to a long wait in the line. Some dhaba provide an extra chair for the customer to wait. But after waiting too long, they leave. However, waiting chairs alone would not solve a problem when customers withdraw and go to the next dhaba. This causes a lot of loss to our dhaba. This shows a need of a numerical model for the restaurant management to understand the situation better. This paper aims to show that queuing theory satisfies the model when tested with a real-case scenario. The authors obtained the data from a "Gulshan Dhaba" in Hodal, Haryana. To derive the arrival rate, service rate, utilization rate, waiting time in queue, and potential customers' probability to balk. The collected data is analysed by using Little's Theorem and M/M/1 queuing model. The arrival rate at "Gulshan Dhaba", Hodal during its busiest period of the day is two customers per minute (cpm), while the service rate is $2.02 \mathrm{cpm}$ during our study period. The average number of customers in the restaurant is 100 , and the utilization period is 0.99 .
\end{abstract}

Keywords: Queuing theory; Little's Theorem; Kendall's notation; Waiting Lines and Dhaba

\section{Introduction}

With the growth of population and living standards, people are keen to take food in dhaba/restaurant. Visitors, tourists, office goers take regular food in a restaurant within their budget. Dhaba services by providing food, breakfast, tiffin, lunch, dinner, and cold drinks and snacks are top-rated. Nowadays, visitors, customers, tourists, floating people prefer to sit in the restaurant for a while and take necessary appetizers and go away [1]. Thus, dhaba can be planned to set up in outer of town/city of thick population, on the high way side. The more option for food is kept to serve, the more the dhaba will be famous. There are several determining factors for a dhaba to be considered a good or a bad one.

Taste, cleanliness, the restaurant layout and settings are some of the most critical factors. These factors, when managed carefully, will be able to attract plenty of customers. However, another factor needs to be considered, especially when the restaurant has already succeeded in attracting customers. This factor is the customers queuing time [2]. In a waiting line system, managers must decide what level of service to offer. A low level of service may be inexpensive, at least in the short run, but may incur high costs of customer dissatisfaction, such as lost future business and actual processing costs of complaints [3]. The arrangement of at least 60 persons at a time is considered in the scheme. If the service and quality of food are correctly maintained, then we can take most profit in business. Waiting lines are the most frequently encountered problems in everyday life [4]. A long waiting line may result in loss of customers to an organization. Waiting time can be reduced by providing additional service facilities, but it may increase the idle time of the service mechanism.

Queuing theory is the study of a queue or waiting lines. Some of the analyses that can be derived using queuing theory include the expected waiting time in the queue, the average time in the system, the expected queue length, the expected number of customers served at one time, the probability of balking customers, as well as the probability of the system to be in certain states, such as empty or full [5]. Hence, queuing theory is suitable to be applied in a dhaba

*Corresponding Author - E- mail: Kajalgoyal451@gmail.com 
setting since it has an associated queue or waiting line where customers who cannot be served immediately have to queue for service. This paper uses queuing theory to study the waiting lines in "Gulshan Dhaba" at Hodal, Haryana, India, and the dhaba provides 50 tables out of which some have four chairs, and some have six chairs. Twenty waiters are working at any one time. On a daily basis, it serves over 700 customers during weekdays and over 1200 customers during the weekend. This paper seeks to illustrate the usefulness of applying queuing theory in a real case situation.

\section{Background Of Queuing Theory}

The first paper on queuing theory, "The Theory of Probabilities and Telephone Conversations", was published in 1909 by A.K. Erlang, now considered the father of the field. His work with the Copenhagen Telephone Company is what prompted his initial foray into the field. He pondered the problem of determining how many telephone circuits were necessary to provide phone service that would prevent customers from waiting too long for an available circuit. In developing a solution to this problem, he realised that minimizing waiting time was applicable to many fields and further developed the theory. He identified that the number of telephone conversations and telephone holding time fit into Poisson distribution and exponentially distributed.

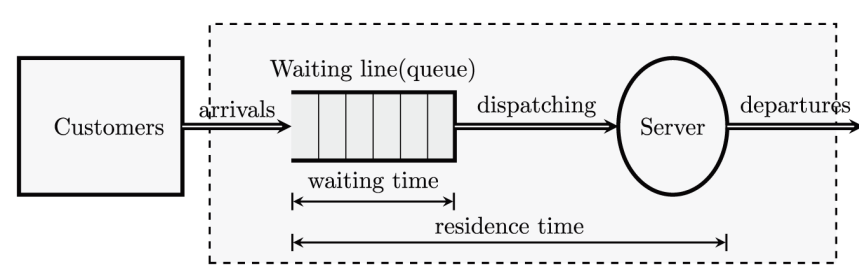

Fig. 1 Queuing System

The mechanism of a queuing process is very simple. Customers arrive at the service counter and are attended by one or more of the servers. As soon as a customer is served, he departs from the system.

\section{Queuing Model}

It is a suitable model used to represent a service-oriented problem, where customers arrive randomly to receive some service, the service time being also a random variable. The principal actors in a queuing situation are the customer and the server. On arrival at a service facility, they can start service quickly or wait in a queue if the server is busy. From the analysing queues, the arrival of customers is represented by the inter-arrival time between successive customers, and the service is described by the service time per customer. Customers may jockey from one queue to another in the hope of reducing waiting time. They may also balk from joining a queue altogether because of long delay, or they may renege from a queue because they have been waiting too long [6].

Queue: A group of items waiting to receive service, including those receiving the service, is known as a queue.

Arrival rate: Arrival is the number of arrivals per unit of time.

Service rate: It is the rate at which the customer is served in the system.

Service time: The time taken by the server to complete service is known as service time.

Server: It is a mechanism through which service is offered. The queuing calculations change depends on whether there is a single server or multiple servers for the queue [7].

Queue Lengths: The queue in a system can be modelled as having infinite or finite queue length. This includes the customers waiting in the queue.

Queue Discipline: It is the order in which the members of the queue are offered service. There are several possibilities in terms of the sequence of customers to be served such as FIFO (First in First Out), random order, LIFO (Last in First Out), SIRO (Service in Random Order) [8]

Waiting time in a queue: Time spent by a customer in the queue before being served.

Waiting time in the system: It is the total time spent by a customer in the system.

It can be calculated as follows:

Waiting time in the system=Waiting time in queue + Service time

In this section, we will discuss two common concepts in queuing theorem.

\section{Little's Theorem}

It describes the relationship between average queueing length, average arrival rate and average waiting time in a queue. The significant part of this theorem is that it is valid for any queueing system with a 
steady state condition. Massachusetts Institute of Technology (MIT) professor John Little developed Little's law in 1954.

The theorem states that the expected number of customers $(\mathrm{N})$ for a system in steady state can be determined using the following equation:

$$
\mathbf{L}=\lambda \mathbf{t} \text {. }
$$

The average number in the system $=$ Arrival rate $\times$ Average system time. Here, $\lambda$ is the average customer arrival rate, and $\mathrm{T}$ is the average service time for a customer. Consider the example of a Dhaba, where the customer's arrival rate $(\lambda)$ doubles, but the customers still spend the same time in the dhaba(T). These facts will double the number of customers in the dhaba (L). Little's theorem is helpful in quantifying the maximum achievable operational improvements and estimating the performance change when the system is modified.

\section{Kendall's Notation}

Kendall's Notation is a system of notation according to which the various characteristics of a queuing model are identified. Kendall (Kendall, 1951) has introduced a set of notations that have become standard in the literature of queuing models.

A general queuing system is denoted by

$(\mathrm{A} / \mathrm{B} / \mathrm{C}):(\mathrm{D} / \mathrm{E})$

Where $\mathrm{A}$ is the probability distribution of interarrival time, $\mathrm{B}$ is the probability distribution of service time, $\mathrm{C}$ is number of servers in the system, $\mathrm{D}$ is the maximum number of customers allowed in the system, $\mathrm{E}$ is queue discipline.

\section{Gulshan Dhaba Queuing Model}

Queuing models are also called waiting line models. We are quite familiar with queues in our day-today life. Common examples of queuing models that we encounter are going to a doctor or going to a barbershop. Queuing models can be of several types. The first category is called a single server queuing model, where there is only one server. We also have multiple server queuing models where there are multiple servers for the same job. The data were obtained from Gulshan dhaba through interviews with the dhaba manager as well as data collections through observations at the dhaba. The daily number of visitors was obtained from the dhaba itself. The dhaba has been recording the data as part of its end of day routine. We also interviewed the dhaba manager to find out about the capacity of the dhaba, the number of waiters and waitresses, and the number of chefs in the dhaba. Based on the interview with the restaurant manager, we concluded that the queuing model that best illustrates the operation of Gulshan dhaba is $\mathrm{M} / \mathrm{M} / 1$. This is a queuing model in which the arrival is Marcovian, and departure distribution is also Marcovian, number of servers is one. In our observation, the dhaba has several waitresses, but they only have one chef to serve all of the customers in the actual waiting queue.

For the analysis of the Gulshan dhaba M/M/1 queuing model, the following variables will be investigated: -

Probability of zero customers in the dhaba: $P_{0}=1-\rho$

Pn: The probability of having $\mathrm{n}$ customers in the dhaba $\operatorname{Pn}=(1-\rho) \rho^{\mathrm{n}}$

Ls: average number of customers dining in the dhaba $\lambda /(\mu-\lambda)$

Lq: average number in the queue.

$$
\lambda^{2} /(\mu-\lambda)
$$

Ws: average time spent in Gulshan dhaba, including the waiting time.

$$
1 /(\mu-\lambda)
$$

Wq: average waiting time in the queue.

$$
\lambda / \mu(\mu-\lambda)=\mathrm{Lq} / \lambda
$$

Here, $\lambda$ : Arrival rate

$$
\mu \text { : Service rate }
$$

$\rho: \lambda / \mu$ utilization rate

\section{Result And Duscussion}

It can be understood from the table 1 that the number of customers on Saturdays and Sundays are double the number of customers during weekdays. The busiest period for the dhaba is on the weekend during dinner time. Hence, we will focus our analysis in this time window.

Table 1 Number of customer visited

\begin{tabular}{cccccccc}
\hline Day/Week & Mon & Tue & Wed & Thu & Fri & Sat & Sun \\
\hline $1^{\text {st }}$ week & 450 & 495 & 420 & 476 & 495 & 920 & 1020 \\
$2^{\text {nd }}$ week & 415 & 470 & 460 & 419 & 510 & 905 & 990 \\
$3^{\text {rd }}$ week & 490 & 450 & 415 & 438 & 530 & 950 & 960 \\
$4^{\text {th }}$ week & 500 & 520 & 490 & 498 & 560 & 980 & 1000 \\
\hline
\end{tabular}

Our teams conducted the research at dinner time. On average, 360 people are coming to the dhaba in 3 hours' time window of dinner time.

From this we can derive the arrival rate as: $\lambda=360 / 180$

$\lambda=2$ customer/hour 
We also found out from observation and discussion with the manager that each customer spends 50 minutes on average in the $\operatorname{dhaba}(\mathrm{W})$. The queue length is around 30 people (Lq) on average, and the waiting time is around 13 minutes. It can be seen that the observed actual waiting time does not differ by much when compared to the theoretical waiting time as shown below.

$$
\mathrm{Wq}=\mathrm{Lq} / \lambda=30 / 2=15
$$

Next, we will calculate the average number of people in the dhaba using

$\mathrm{L}=50 * 2=100$ customers

Having calculated the average number of customers in the dhaba, we can also derive the utilization rate and the service rate

$\mu=\lambda(1+\mathrm{L}) / \mathrm{L}$

$=2(1+100) / 100$

$=2.0$

Hence, $\rho=\lambda / \mu=2 / 2.02=0.99$

With the very high utilization rate of 0.99during dinner time, the probability of zero customers in the dhaba is minimal, as can be derived

$$
\begin{aligned}
P_{0} & =1-\rho \\
& =1-0.99=0.01
\end{aligned}
$$

The generic formula that can be used to calculate the probability of having $\mathrm{n}$ customers in the dhaba is as follows:

$$
\begin{aligned}
& P n=(1-\rho) \rho^{n} \\
= & 0.01 *(0.99)^{n}
\end{aligned}
$$

We assume that potential customers will start to balk when they see more than 25 people are already queuing for the dhaba. We also assume that the maximum queue length that a potential customer can tolerate is 35 people. As the capacity of the dhaba when fully occupied is 90 people, we can calculate the probability of 25 people in the queue as the probability when there are 125 people in the system (100 in the dhaba and 25 or more queuing) as follows:

Probability of customers going away $=\mathrm{P}$ $($ more than 25 people in the queue $)=\mathrm{P}($ more than 125$)$ people in the dhaba)

$$
\begin{aligned}
\mathrm{P}_{100-125} & =\sum_{\mathrm{n}=100}{ }^{125}(0.01)(0.99)^{\mathrm{n}} \\
& =8.417 \%
\end{aligned}
$$

\section{Evaluation}

i. The utilization is directly proportional to the mean number of customers. It means that the mean number of customers will increase as the utilization increases

ii. 2)A bored customer is an impatient customer; activity speeds the perception of time.

iii. 3)The utilization rate at the dhaba is very high at 0.99 . On weekdays, the utilization rate is almost half of it. This is because the number of visitors on weekdays is only half of the number of visitors on weekends

iv. 4) People love to eat out, and they like to do so in a dhaba that is comfortable, safe and enjoyable. Food isn't the only thing that matters; a dhaba interior design also helps draw customers in and entices them to come back again and again.

v. If the customer's waiting time is lower or we waited for less than 25 minutes, the number of customers that can be served per minute will increase. When the service rate is higher, the utilization will be lower, making the probability of the customers going away decrease.

\section{Benefits}

i. Queuing theory is applied to dhaba managers looking for ways to improve their customers' comfort and satisfaction while they wait for service.

ii. This research can help Gulshan Dhaba increase its QoS (Quality of Service) by anticipating many customers in the queue.

iii. A goal of using queuing theory for restaurant analysis is to improve customer wait times. This might be accomplished by adding more servers at certain times of the day, on busier days or after special events taking place in the area. If it's not possible to speed up service, you also can work to improve the waiting experience.

iv. By anticipating the huge number of customers coming and going in a day, the dhaba can set a target profit that should be achieved daily.

v. The formulae used during the completion of the research are applicable for future research and could be used to develop more complex theories. 


\section{Conclusion}

This research paper has discussed the application of queueing theory of "Gulshan Dhaba".Restaurants create a nice, clean and comfortable environment where patrons can enjoy their food. The food is hygienically prepared and presented as compared to dhaba. But the dhabas bring nature to the patrons. The atmosphere here is usually all-natural. Seating may not be comfortable, and is usually rudimentary. The environment of the kitchen is questionable. Food is either simple home-style (genuine truck-stop dhabas) or robust. But the food is better than a restaurant. But the service quality is not good. So, we want to improve the service quality of dhabas in this paper. From the result we have obtained, the rate at which customers arrive in the queuing system is two customers per minute, and the service rate is 2.02 customers per minute. The probability of buffer flow, if there are 25 or more customers in the queue, is 30 out of 100 potential customers. The probability of buffer overflow is the probability that customers will run away because maybe they are impatient to wait in the queue. It can be concluded that the arrival rate will be lesser, and the service rate will be greater if it is on weekdays since the average number of customers is less as compared to those on weekends. We hope that this research can contribute to the betterment of Gulshan Dhaba in terms of its way of dealing with customers.

\section{References}

1. Umesh Sharma and Kajal Garg, (2021) “A Queuing Model for Improving the Management of Oxygen Cylinder in COVID-19." International Journal of Innovative Science and Research Technology, Vol.2, pp.729-731

2. Nityangini Jhalaland Pravin Bhathawala, (2017) "Analysis and Application of Queuing Theory in "Analysismarkets", International Journal of Innovative Research in Science, Engineering and Technology, Vol.6, pp.17974-17979

3. Umesh Sharma; Kajal Garg (2021)" Impact Of Queuing Theory in Whole Process in Business from Manufacture to Consume Authors," International Journal of Innovative Research in Technology, pp. 297-300.

4. Nityangini Jhalal and Pravin Bhathawala, (2016), "Application of Queuing Theory in Banking Sector, International Organization of Scientific Research-Journal of Mathematics, Vol.12, pp.73-75

5. Priyansh and Umesh Sharma,(2021) "Queuing Theory Applied in Professional Life", International Journal of Research and Analytical Reviews, pp.778-783.

6. Nityangini Jhalal and Pravin Bhathawala, (2017) "Mathematical Analysis of Multi-phase single server and multi-phase multi server: Comparison study", International multi-phase multi server: Comparison study", International
Journal of Mathematics Trends and Technology, Vol. 51, pp.354-357

7. Nityangini Jhalal and Pravin Bhathawala, (2016)," Smart Queue management system for banking sector", International Journal of Business Management and Economics Research, Vol. 3, pp.29-34

8. Nityangini Jhalal and Pravin Bhathawala, (2017) Application of Queuing Theory to Airport related problems, Global Journal of Pure and Applied mathematics, Global Journal of Pure and Applied mathematics, Vol. 13, pp.3863-3868. 